07,08

\title{
Механизм обусловленного динамической примесной подсистемой аномального поведения пластического течения материалов с высоким кристаллическим рельефом
}

\author{
(C) Б.В. Петухов \\ Институт кристаллографии им. А.В. Шубникова ФНИЦ „Кристаллография и фотоника“ РАН, \\ Москва, Россия \\ E-mail: petukhov@ns.crys.ras.ru
}

Поступила в Редакцию 3 июля 2021 г.

В окончательной редакции 3 июля 2021 г.

Принята к публикации 29 августа 2021 г.

\begin{abstract}
Развивается модель динамического взаимодействия дислокаций с примесной подсистемой кристаллов с высоким потенциальным рельефом кристаллической решетки (барьерами Пайерлса). К числу таких материалов относятся металлы с объемно-центрированной кубической структурой, полупроводники, керамики и многие другие. Показано, что модификация примесных миграционных барьеров вблизи дислокационного ядра существенно влияет на сегрегацию примесей на движущейся дислокации. Обосновывается наличие существенно неравновесной начальной стадии кинетики сегрегации, приводящей к аномалиям дислокационной динамики и предела текучести материалов.
\end{abstract}

Ключевые слова: динамика дислокаций, сегрегация примесей в дислокационном ядре, синергетика дислокационной и примесной подсистем, устойчивые и неустойчивые режимы, аномалии пластического течения.

DOI: 10.21883/FTT.2021.12.51674.157

\section{1. Введение}

При исследовании или практическом использовании кристаллических материалов выявляется ряд аномальных режимов поведения их механических свойств. В исходно мало дефектных кристаллах неустойчивости пластического течения могут возникать, как показано в работе [1], в результате катастрофического размножения дислокаций с образования волн их плотности. В материалах, содержащих заметное количество примесей, возможен другой механизм аномального поведения, обусловленный динамическим взаимодействием дислокационной и примесной подсистем. Влияние примесей на динамику дислокаций существенно модифицирует механические свойства кристаллических материалов и является предметом всестороннего изучения, как экспериментального, так и теоретического [2-5]. Выявляют несколько динамических режимов в различных областях значений температуры и скоростей деформации. Один отвечает статичным примесям, как бы „вмороженным“ в кристаллическую решетку и создающим индивидуальные препятствия для движения дислокаций. При других условиях примеси диффундируют к дислокациям и образуют вокруг них обогащенные атмосферы, модифицирующие динамические характеристики дислокаций своим коллективным влиянием, приводя к их так называемому „старению“. Возможно также силовое увлечение дислокациями малоподвижных примесей [6]. На макроскопическом уровне это вызывает „динамическое деформационное старение“ материала в целом. При сравнимых подвижностях дислокаций и примесей нередко возникает нестабильность пластического течения, проявляющаяся в виде скачков на деформационных кривых (эффект Портевена-Ле Шателье [7-11]). Еще один режим отвечает высоко подвижным примесям, атмосфера которых легко сопровождает перемещение дислокаций, не создавая большого дополнительного торможения [12].

Впервые взаимодействие примесей с равномерно движущейся дислокацией краевого типа рассматривалось в работе [13] и затем в [14-19] и многих других. В этих работах рассчитывалось образование вокруг дислокации континуального примесного облака, называемого атмосферой Коттрела. К настоящему времени динамическое взаимодействие примесей с дислокациями хорошо изучено для материалов с гране-центрированной кубической (ГЦК) структурой кристаллической решетки. Значительно менее изучена ситуация для кристаллов с объемно-центрированной кубической (ОЦК) структурой, полупроводников, керамик и других материалов, в которых движение дислокаций лимитируется высоким потенциальным рельефом кристаллической решетки, называемым также барьерами Пайерлса. Закономерности динамического старения дислокаций зависят от механизма их движения и различны для двух указанных типов материалов. В материалах с ГЦК структурой кристаллический рельеф низок, и дислокации движутся по мере преодоления барьеров, созданных „дислокациями леса“ или какими-либо локальными центрами. В материалах с высоким кристаллическим рельефом имеет место так называемый кинковый механизм движения дислокаций [20], и кинетика вносимого движущимися дислока- 


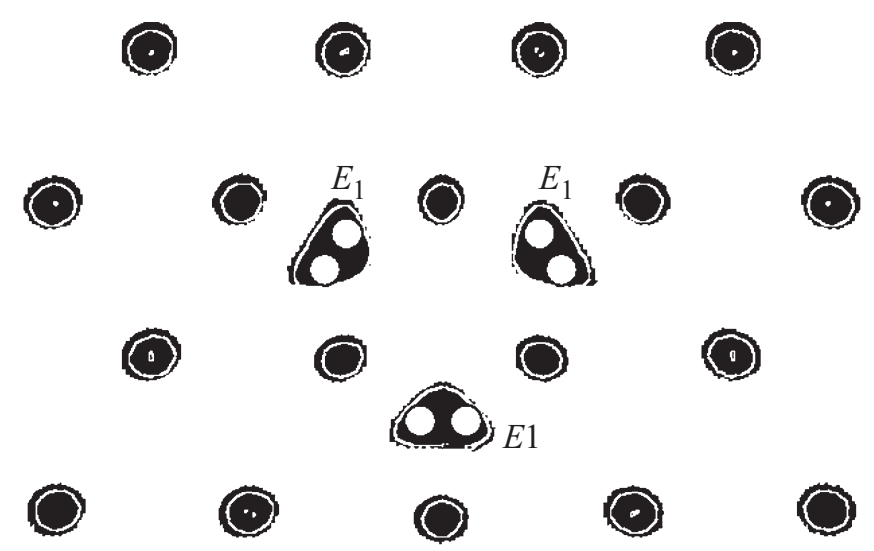

Рис. 1. Расположение узлов $E_{1}$ с максимальной энергией связи примесных атомов Н с винтовой дислокацией в $W$.

циями возмущения примесной подсистемы совершенно иная [21]. В таких материалах помимо образования континуальной коттреловской атмосферы возможны также эффекты, обусловленные перераспределением примесей на короткой шкале вблизи ядра дислокации. Например, в работе [22] изучается ситуация, когда динамическое старение в ниобии с кислородом в температурном интервале 423-448 K обусловлено перераспределением примесей на межатомных расстояниях вблизи ядра дислокации, которое авторы называли динамическим старением по Снуку (Snoek).

При традиционном континуальном подходе зачастую пренебрегают взаимодействием примесей с винтовыми дислокациями, поскольку те не создают дальнодействующего поля гидростатического давления. Однако при этом не учитываются многие другие типы взаимодействия, имеющие место на межатомной шкале в дислокационных ядрах, например, химической природы. В противоположность этому атомистичекие расчеты выявляют наличие достаточно большой энергии связи примесей с винтовыми дислокациями. Так, например, в работе [23] рассчитывалось взаимодействие примесей водорода с винтовой дислокацией в $W$ в различных позициях и для наиболее благоприятной позиции получена довольно значительная энергия связи равная $256 \pm 32 \mathrm{meV}$. Шесть таких эквивалентных позиций, обозначаемых $E_{1}$, изображены на рис. 1.

Для расчета эффектов, связанных с короткодействием, континуальный подход неприменим, и необходимо дискретное описание. Нередко считается [24,25], что это может быть сделано только путем численного моделирования вследствие больших трудностей в описании ко-эволюции двух многочастичных систем: растворенных атомов и дислокаций. В полном объеме это действительно так, однако, если ставить менее амбициозную задачу качественного выяснения механизмов явлений и тенденций, связанных с влиянием различных материальных параметров, может быть полезен упрощенный подход, излагаемый в настоящей работе.
Ключевым вопросом теории динамического деформационного старения материалов является вариация содержания примесей на дислокационных линиях. Однако этот вопрос в имеющихся работах освещается недостаточно последовательно и нередко просто постулируется кинетический закон в виде [19]:

$$
c\left(t_{a}\right)=c_{0}+\left(c_{e q}-c_{0}\right)\left\{1-\exp \left[-\left(t_{a} / \tau_{D}\right)^{n}\right]\right\} .
$$

Здесь $c_{0}-$ средняя концентрация примесей в объеме кристалла, $c_{e q}-$ равновесная концентрация примесей в дислокационном ядре, $t_{a}$ - время, проводимое дислокацией перед барьером в ожидании перехода, равное $h / V$, $h$ - расстояние между барьерами (для барьеров Пайерлса $h$ есть период кристаллической решетки $a), V-$ средняя скорость дислокации, $\tau_{D}-$ характерный временной масштаб релаксации примесной подсистемы, показатель степени $n$ изменяется в пределах $1 / 3 \div 1$. Уравнение $(1)$ представляет собой простую экстраполяцию между начальной степенной кинетикой сегрегации примесей на дислокационной линии и финальным выходом на равновесие.

Но в ряде случаев наблюдается рост концентрации примесей на дислокациях при увеличении скорости их движения, что противоречит закону (1). Такое поведение свидетельствует о существенном дополнении диффузионных процессов непосредственным увлечением примесей ядром дислокации. Кроме того, для лучшего понимания явления было бы полезно не постулировать формулы типа (1), а вывести кинетический закон из лежащих в основе физических механизмов хотя бы в рамках простой модели. Развитие соответствующей теории и является целью настоящей работы.

\section{2. Модель короткодействующих примесей}

При различных скоростях движения дислокаций в сравнении с подвижностью примесей проявляются различные пространственные масштабы их взаимодействия. В изучаемом здесь случае достаточно быстрого движения оказываются существенны малые масштабы порядка нескольких периодов кристаллической решетки вокруг дислокации. Особую важность короткодействующему взаимодействию с примесями в масштабе дислокационного ядра придает дискретность движения дислокаций на атомной шкале - между долинами периодической кристаллической решетки - происходящего, как известно, термически активируемыми скачками [20]. Скачкообразное изменение положения дислокации приводит к перераспределению примесной атмосферы на каждом шаге, кинетика которого зависит от устройства потенциального рельефа для миграции примесей в окрестности дислокационного ядра. Для полного количественного описания подобных процессов микроскопическая картина, безусловно, важна, однако для выяснения механизма явления полезно максимально абстрагироваться от деталей и сконцентрироваться на его универсальных чертах. 


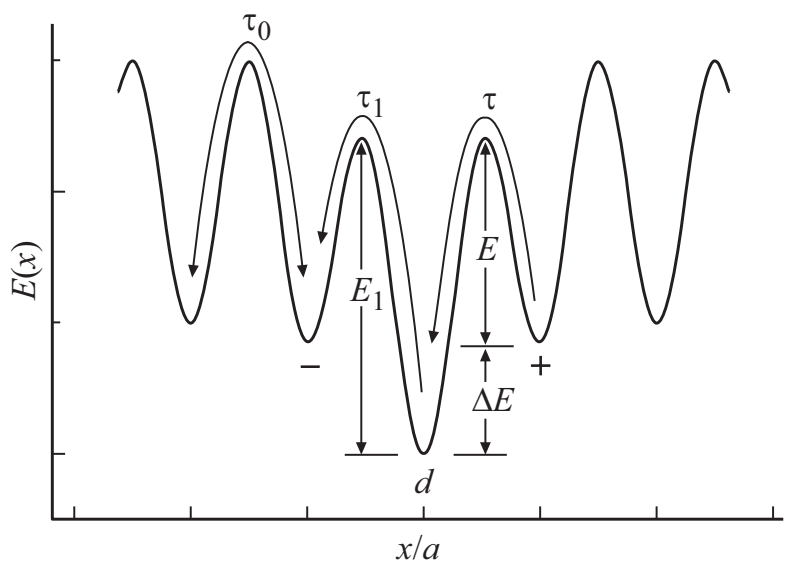

Рис. 2. Схема миграционного рельефа примесей вблизи ядра дислокации. Выделены состояния непосредственно в ядре $(d)$ и соседние предыдущее $(-)$ и последующее $(+)$ с указанием времен переходов примесей между этими состояниями и объемом кристалла.

Схематически простейший одномерный вид миграционного рельефа изображен на рис. 2. Под рассматриваемыми состояниями примесного содержания в ядре и вокруг ядра, как это для наглядности иллюстрируется на рис. 1 тремя состояниями, в действительности могут пониматься любые совокупности эквивалентных ячеек, заполняемые примесями.

Вдали от дислокации миграционный рельеф периодичен, и время $\tau_{0}$ смещения примеси на период решетки имеет невозмущенное значение. В окрестности ядра потенциальный рельеф искажен взаимодействием, так что имеется глубокий минимум, отвечающий наиболее энергетически выгодному расположению примеси относительно дислокации, отмеченный на рисунке буквой $d$. Притяжение к этому положению понижает барьер $E$ для перехода примеси в него и уменьшает время соответствующего термоактивируемого скачка $\tau=\tau_{*} \exp (E / k T)$ по сравнению со временем обратного перехода $\tau_{1}=\tau_{* 1} \exp \left(E_{1} / k T\right)$ или временем миграции вдали от ядра $\tau_{0}=\tau_{* 0} \exp \left(E_{0} / k T\right)$, так что $\tau \ll \tau_{0}, \tau_{1}$. Здесь $\tau, \tau_{1}, \tau_{0}$ - предэкспоненциальные множители, принимаемые постоянными. Смысл энергий активации $E_{0}, E_{1}, E=E_{1}-\Delta E$, поясняется на рис. 2. Существование пониженного барьера $E$ для перехода примесей в ядро дислокации по сравнению с барьерами переходов $E_{0}$ в объеме кристалла приводит к необходимости выделения процессов перераспределения примесей вокруг ядра в особый класс и требует отдельного рассмотрения, как это отмечалось в $[21,22]$. Похожий подход применялся в работах $[23,24]$, в которых принималось во внимание только перераспределение примесных атомов водорода между ближайшими междоузлиями при перемещении дислокации в $\alpha$-Fe без включения новых примесей. Таким образом, общее число примесей в окружении ядра считалось постоянным, в отличие от настоящей работы, где будет учитываться также включение в игру новых примесей и рост их содержания в ядре по мере перемещения дислокации.

Примеси, попадающие в наиболее низкое по энергии состояние $d$, труднее всего оторвать от дислокации и, следовательно, они представляют главный интерес для вычисления силы пиннинга. По этой причине основное внимание будет уделено расчету заполнения состояния $d$, а прочие состояния в простейшем приближении будут считаться слабо возмущенными и трактоваться как некий резервуар с постоянной концентрацией $c_{0}$. Исключение составляют смежные с $d$ состояния в процессе перемещения,,+- поскольку вследствие движения дислокации с возможным увлечением или отставанием примесей между этими состояниями происходит существенное перераспределение примесной концентрации. Так как считается, что дислокации движутся с достаточно большой скоростью $V$, время $t_{a}=a / V$, проводимое в одной долине кристаллического рельефа, в рассматриваемой упрощенной модели предполагается недостаточным для включения в игру примесей из более удаленных узлов кристаллической решетки.

Изучим эволюцию примесного содержания в ядре дислокации, характеризуемого концентрацией $c_{d}$ в расчете на один период решетки вдоль дислокационной линии. Введем также обозначение $c$ для концентрации примесей в примыкающих состояниях. Считается, что движение дислокаций имеет циклический характер и подавляющую часть времени цикла $t_{a}$ занимает время ожидания перед барьером. Временем распространения кинков на весь дислокационный сегмент после их зарождения будем пренебрегать. Из-за большой разницы времен перехода примесей в ядро дислокации $\tau_{*}$ и в соседние узлы решетки $\tau_{* 1}, \tau_{* 0}$ эволюция примесного содержания в одном цикле происходит в две существенно отличающиеся по длительности стадии. В течение времени порядка $\tau_{*}$ происходит только самый быстрый процесс перехода примесей из ближайших состояний $( \pm)$ в ядро $(d)$ практически без участия более удаленных примесей. И лишь впоследствии за время, включающее $\tau_{* 0}$, происходит обмен с примесями из резервуара и устанавливается равновесное содержание. Если время ожидания перехода дислокации через барьер $t_{a}$ относительно невелико, равновесное содержание не достигается и количество сегрегированных примесей имеет существенно неравновесный характер. Особый интерес для изучения условий возникновения аномальных режимов дислокационной динамики представляет поведение на начальной стадии, для которого приведем аналитическое описание.

Запишем уравнение для перераспределения примесных концентраций в течение времени $t_{a}$, когда дислокация покоится в одной из долин кристаллического рельефа в ожидании скачка в следующую долину

$$
\frac{d c_{d}}{d t}=c\left(1-c_{d}\right) / \tau \text {. }
$$

Правая часть уравнения (2) описывает поток примесей из примыкающих состояний в ядро $(d)$ в единицу времени. Принимается во внимание, что переходы возможны 
лишь в незанятые состояния, так что, например, частота переходов в состояние $d$ пропорциональна вероятности $1-c_{d}$ того, что узел свободен, и т. д. Вследствие сохранения суммарного числа примесей $c_{d}+c$ в течение одного цикла эта сумма равна своему начальному значению $c_{d}+c=c_{d 0}+c_{c 0}$, что позволяет исключить из (2) $c$. Получаемое замкнутое уравнение

$$
\frac{d c_{d}}{d t}=\left(1-c_{d}\right)\left[-c_{d}+c_{d 0}+c_{c 0}\right] / \tau
$$

легко решается путем разделения переменных

$c_{d}(t)=\frac{\left(c_{d 0}+c_{c 0}\right)\left(1-c_{d 0}\right) \exp \left[\left(1-c_{d 0}-c_{c 0}\right) t / \tau\right]-c_{c 0}}{\left(1-c_{d 0}\right) \exp \left[\left(1-c_{d 0}-c_{c 0}\right) t / \tau\right]-c_{c 0}}$.

\section{3. Увлечение примесей при стационарном движении дислокаций}

Стационарное с макроскопической точки зрения движение дислокации по периодическому рельефу кристаллической решетки на микроуровне осуществляется последовательными термоактивируемыми скачками на один период решетки с временем ожидания одного скачка $t_{a}$, так что средняя скорость дислокации есть $V=a / t_{a}$. Перемена положения дислокации сопровождается соответствующей перестройкой примесной подсистемы. При скачке дислокации через барьер она попадает в новое состояние с невозмущенной концентрацией $c_{0}$, а набранные ей к моменту перескока примеси $c_{d}\left(t_{a}\right)$ сбрасываются. Примеси, оставленные в покинутой дислокацией долине кристаллического рельефа, стремятся релаксировать к новому положению дислокации. При этом величины примесного содержания, достигнутые на предыдущем шаге к моменту скачка дислокации $t_{a}$, будут играть роль начальных условий для релаксации концентраций на новом этапе $c_{c 0}^{(2)}=c_{d}^{(1)}\left(t_{a}\right)$. Решением уравнения (2) на новом этапе будет

$$
\begin{aligned}
& c_{d}^{(2)}(t) \\
& =\frac{\left(c_{d}^{(1)}\left(t_{a}\right)+c_{0}\right)\left(1-c_{0}\right) \exp \left[\left(1-c_{d}^{(1)}\left(t_{a}\right)-c_{0}\right) t / \tau\right]-c_{d}^{(1)}\left(t_{a}\right)}{\left(1-c_{0}\right) \exp \left[\left(1-c_{d}^{(1)}\left(t_{a}\right)-c_{0}\right) t / \tau\right]-c_{d}^{(1)}\left(t_{a}\right)} .
\end{aligned}
$$

Это выражение совместно с новыми начальными условиями дает рекуррентное соотношение для описания ко-эволюции дислокации и примесной подсистемы.

При описании движения дислокации как серии последовательных скачков будем пренебрегать статистическим разбросом времен переходов $t_{a}$, что до некоторой степени оправдано регулярностью барьеров, создаваемых периодическим кристаллическим рельефом. Эволюция кинетических зависимостей $c_{d}(t)$ для нескольких последовательных шагов, начиная от „свежей“ дислокации (то есть не имеющей избыточного количества примесей

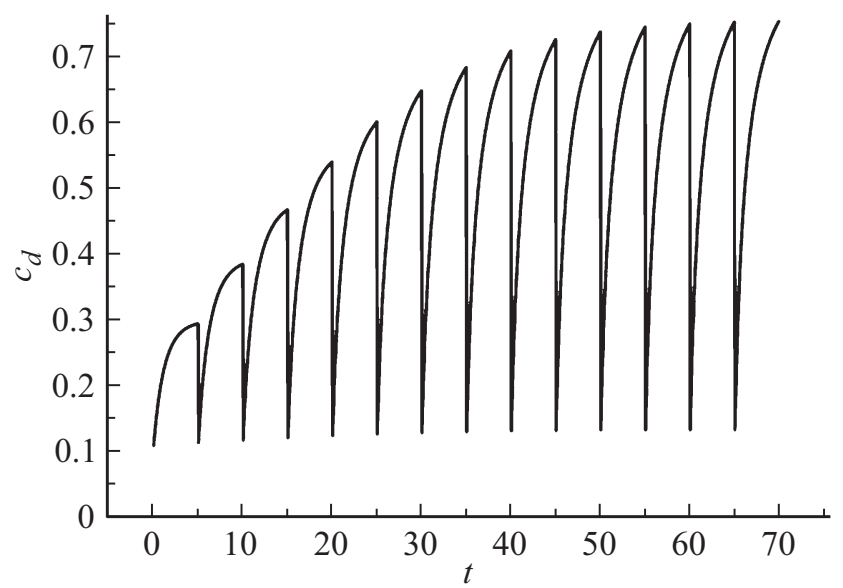

Рис. 3. Эволюция содержания примесей в дислокационном ядре для серии 12 переходов первоначально „свежей“ дислокации на период решетки при $c_{0}=0.1, t_{a}=5 \tau$.

по сравнению со средней их концентрацией в объеме кристалла $c_{0}$ ), проиллюстрирована на рис. 3. Как видно из рисунка, имеется тенденция к установлению некоторой предельной самовоспроизводящейся кинетической зависимости $c_{d}^{s}(t)$.

В масштабе времени большом по сравнению с временем $t_{a}$ элементарного перехода движение дислокации при воспроизводящейся кинетической зависимости $c_{d}^{s}(t)$ является стационарным (и будет таким называться в дальнейшем в указанном понимании). Содержание примесей при таком стационарном движении дислокации будет характеризоваться амплитудным значением $c_{d}^{s}(t)$ в интервале $\left(0, t_{a}\right)$, которое обозначим как $c_{d a}^{s}$. Устанавливающееся при большом количестве переходов дислокации амплитудное значение примесного содержания в ядре $c_{d a}^{s}$ может быть найдено из уравнения, получающегося из (5) при $t=t_{a}$ и сравнивающихся $c_{d}^{(2)}\left(t_{a}\right)$ и $c_{d}^{(1)}\left(t_{a}\right)$,

$$
t_{a} / \tau=\frac{1}{1-c_{0}-c_{d a}^{s}} \ln \left[\frac{c_{d a}^{s}\left(1-c_{d a}^{s}\right)}{c_{0}\left(1-c_{0}\right)}\right] .
$$

Обращенное выражение (6) является аналогом формулы (1) для случая движения дислокаций с определяющим влиянием кристаллического рельефа. Это выражение позволяет рассчитывать зависимость примесного содержания на дислокации от заданной скорости ее движения $V=a / t_{a}$, средней концентрации примесей в объеме $c_{0}$, температуры $T$ и других параметров. Возможен также другой вариант кинетики дислокационного старения, а именно, при заданной движущей дислокацию силе (или, эквивалентно, напряжении). В этом случае следует выразить скорость движения дислокации через барьеры в зависимости от движущей силы, например, с помощью термоактивационного закона

$$
V=V_{0} \exp \left[-\frac{E_{d 0}-\gamma \sigma_{e f}\left(c_{d}\right)}{k T}\right]
$$



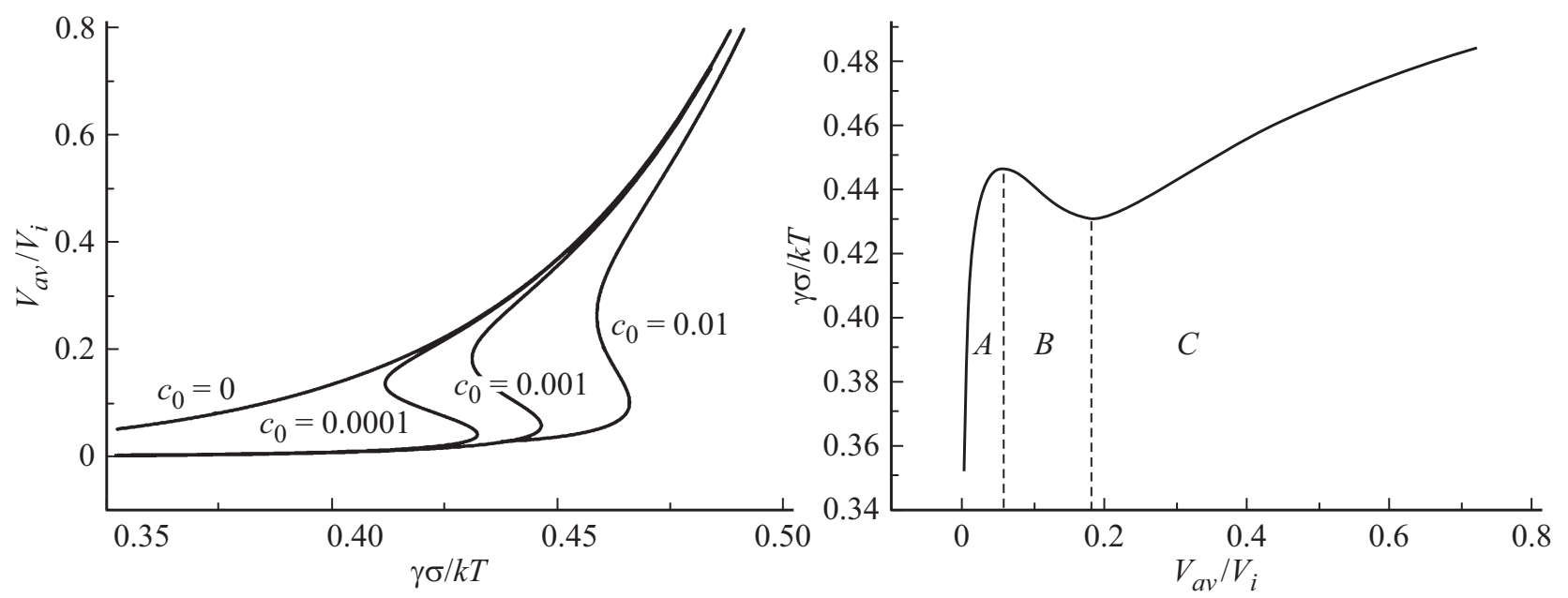

Рис. 4. Зависимость скорости дислокаций, нормированной на $V_{i}=a / \tau$, от напряжения для трех значений средней концентрации примесей (левый рисунок). На правом рисунке кривая, отвечающая объемной концентрации $c_{0}=0.001$, показана в инвертированных осях координат. Значения параметров: $E_{i} / E_{0}=0.5, \gamma \beta / k T=3, a / V_{d 0} \tau_{0}=1$.

Здесь $E_{d 0}$ - энергия активации движения дислокации в беспримесном материале при исчезающее малой нагрузке, $\gamma$ - активационный объем $\sigma_{e f}-$ эффективное напряжение, учитывающее тормозящее влияние сегрегированных на дислокации примесей. В различных случаях энергия активации может по-разному выражаться через концентрацию примесей в ядре дислокации. При низкой концентрации следует учитывать дискретность расположения примесей и их индивидуальное взаимодействие с дислокацией по отдельности, как это делалось в $[23,26,27]$. Это имеет место, когда вносимые одной примесью возмущения дислокационной конфигурации не перекрываются между собой. Протяженность такого возмущения составляет величину порядка размера кинка $d_{k} \sim a\left(G / \sigma_{P}\right)^{1 / 2}[26]$, в типичных ОЦК металлах намного превышающую межатомное расстояние. Здесь $G-$ модуль сдвига, $\sigma_{P}-$ напряжение Пайерлса. В случае, когда среднее расстояние между примесями $c_{d} / a$ меньше $d_{k}$, можно использовать усредненное описание взаимодействия между примесями и дислокациями и характеризовать его некоторым тормозящим напряжением $\sigma_{i}$, пропорциональным избыточной примесной концентрации: $\sigma_{i}=\beta\left(c_{d}-c_{0}\right)$, что и будет приниматься далее. Коэффициент пропорциональности $\beta$ может рассматриваться как некоторый феноменологический параметр. При этом входящее в энергию активации в (7) эффективное напряжение имеет вид $\sigma_{e f}=\sigma-\sigma_{i}=\sigma-\beta\left(c_{d}-c_{0}\right)$, где $\sigma-$ внешнее прикладываемое напряжение. Отметим, что линейность эффективного напряжения по концентрации имеет место до тех пор, пока среднее расстояние между примесями $a / c_{d}$ не сравняется с радиусом их взаимодействия вдоль дислокации. При больших концентрациях энергия зацепления дислокаций за примесную атмосферу рассчитывается в духе задачи о цепочке Изинга $[28,29]$, о чем в настоящей работе речь идти не будет.

Так как $\sigma_{e f}$ зависит от времени, при больших по сравнению с периодом решетки дислокационных пробегах следует вычислять среднюю скорость за цикл от 0 до $t_{a}$

$$
V_{a v}=\frac{1}{t_{a}} V_{0} \int_{0}^{t_{a}} d t \exp \left\{-\frac{E_{d 0}-\gamma\left[\sigma-\beta c_{d}(t)+\beta c_{0}\right]}{k T}\right\}
$$

Будет рассматриваться установившееся движение дислокации. С помощью соотношения (3) можно перейти от интегрирования по времени к интегрированию по концентрации $c_{d}$

$$
V_{a v}=V_{0} \exp \left\{-\frac{E_{d 0}-\gamma\left(\sigma+\beta c_{0}\right)}{k T}\right\} \frac{\tau}{t_{a}} J\left(c_{d a}^{s}\right)=\frac{a}{t_{a}},
$$

где

$$
J\left(c_{d a}^{s}\right)=\int_{c_{0}}^{c_{d a}^{s}} \frac{d c_{d}}{\left(1-c_{d}\right)\left(-c_{d}+c_{a d}^{s}+c_{0}\right)} \exp \left\{-\frac{\gamma \beta c_{d}}{k T}\right\} .
$$

Уравнение, связывающее $c_{d a}^{s}$ с напряжением и температурой, следующее из соотношения (9), есть

$$
\left(\gamma / E_{0}\right) \sigma=1-E_{i} / E_{0}-\left(k T / E_{0}\right) \ln \left[J\left(c_{d a}^{s}\right) V_{0} \tau_{0} / a\right] .
$$

Следует иметь в виду, что $c_{d a}^{s}$ зависит от скорости дислокаций и, следовательно, от напряжения, которое, в результате, должно находиться самосогласованным образом с использованием соотношения (6). Перепишем это соотношение в виде

$$
V_{a v}=\frac{a}{\tau} \frac{1-c_{d a}^{s}-c_{0}}{\ln \left\{\left(1-c_{d a}^{s}\right) c_{d a}^{s} /\left[\left(1-c_{0}\right) c_{0}\right]\right\}} .
$$

Уравнения (10) и (11) дают параметрическое представление силового закона (параметр $c_{d a}^{s}$ ), выражающего зависимость скорости дислокации от напряжения при различных значениях характеристик примесной подсистемы. Это поведение иллюстрируется на рис. 4, $a$. Меняя 


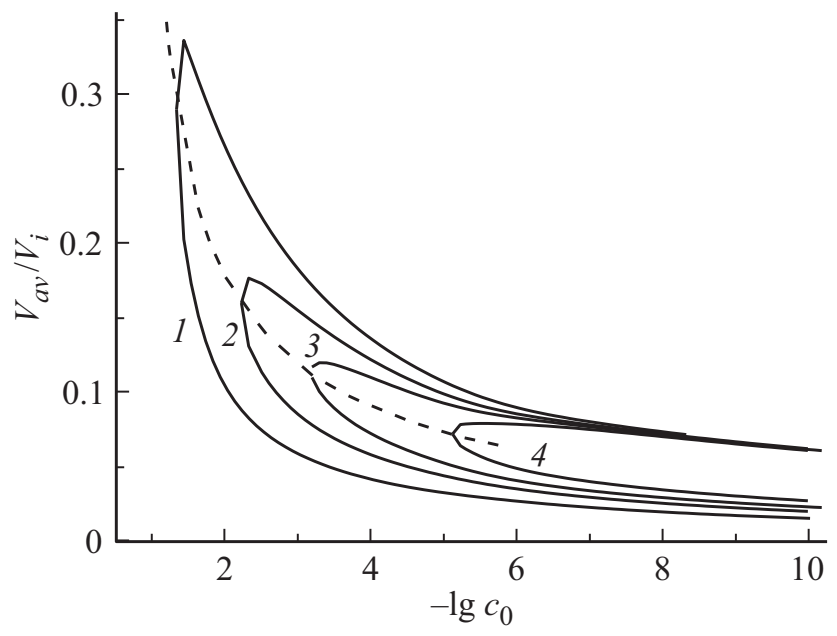

Pис. 5. Границы областей отрицательной скоростной чувствительности дислокационной подвижности при значениях параметров $\gamma \sigma / k T=4(1),=3(2),=2.3$ (3), = 2 (4). Штриховая линия отвечает слиянию трех ветвей решения в одну с исчезновением областей неустойчивости движения дислокаций.

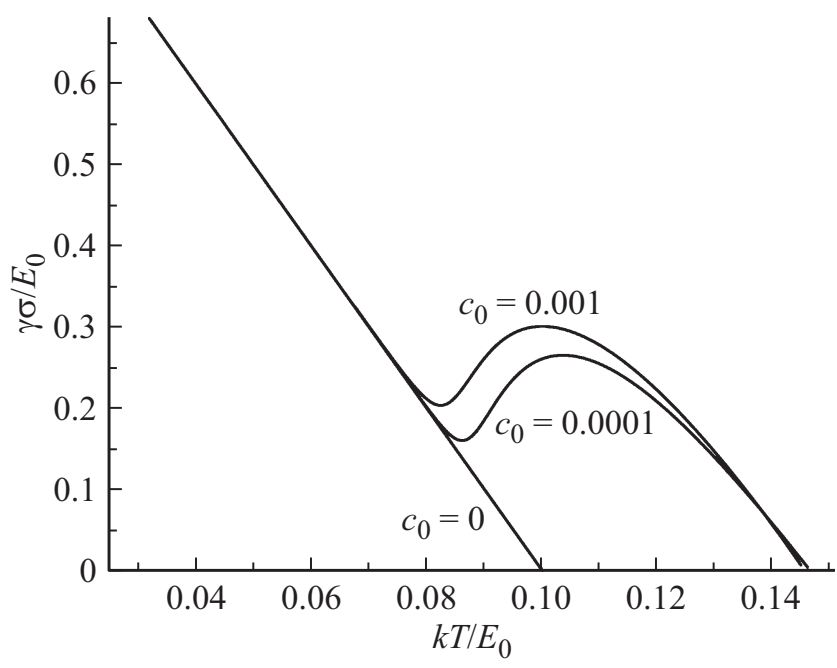

Рис. 6. Температурная зависимость движущей силы, демонстрирующая наличие аномального позитивного поведения в некотором интервале. Значения параметров: $E_{i} / E_{0}=0.7$, $\gamma \beta / E_{0}=0.5$.

местами оси координат, получаем силовой закон, иллюстрирующий существование трех режимов подвижности дислокаций, соответствующих упоминавшимся во введении: режима с упрочняющим влиянием примесей при низкой скорости дислокаций, волочащих свои примесные атмосферы $(A)$, режима с отрицательной скоростной чувствительностью при промежуточных скоростях дислокаций, сопоставимых с подвижностью примесей $(B)$, и режима относительно слабого индивидуального влияния примесей при больших скоростях дислокаций $(C)$ (рис. $4, b)$. Можно видеть, что при высоком напряжении влияние примесей мало, но с понижением напряжения вследствие сегрегации примесей в дислокационном ядре происходит изменение режима движения дислокаций с резким уменьшением их скорости. В некоторой области параметров одному значению напряжения отвечает три решения, или три значения скорости, как это иллюстрируется на рис. 4. В этой области для одного из решений имеет место „отрицательная скоростная чувствительность“, когда скорость дислокации убывает с ростом напряжения. Такое поведение при макроскопических механических испытаниях нередко приводит к нестабильности пластического течения материалов, называемой эффектом Портевена-Ле Шателье [7-11].

На рис. 5 иллюстрируются границы областей существования неустойчивости дислокационной подвижности (границы между областями $A-B$ и $B-C$ рис. 4) при различных средних концентрациях примесей в объеме и температурах.

Динамическое старение дислокаций приводит и к аномалии температурной зависимости напряжения, прикладываемого, чтобы обеспечить заданную скорость движения дислокаций. Эта аномалия проявляется в виде роста напряжения с температурой в некотором температурном интервале, как это иллюстрируется на рис. 6 .

Скоростная чувствительность $d \sigma / d V$ находится с помощью уравнений (10), (11) как

$$
\begin{aligned}
& \frac{d \sigma}{d V}=-\frac{k T}{\gamma J} \frac{d J}{d c_{d a}^{s}} \frac{d c_{d a}^{s}}{d V} \\
& =\frac{k T}{\gamma} \frac{d J}{d c_{d a}^{s}} /\left\{\begin{array}{l}
\ln \left\{\left(1-c_{d a}^{s}\right) c_{d a} /\left[\left(1-c_{0}\right) c_{0}\right]\right\}+ \\
+\left(1-c_{d a}^{s}-c_{0}\right)\left(1-2 c_{d a}^{s}\right) /\left[\left(1-c_{0}\right) c_{0}\right] \\
\ln ^{2}\left\{\left(1-c_{d a}^{s}\right) c_{d a}^{s} /\left[\left(1-c_{0}\right) c_{0}\right]\right\}
\end{array}\right\},
\end{aligned}
$$

где

$$
\begin{aligned}
\frac{d J}{d c_{d a}^{s}}= & \frac{1}{\left(1-c_{0}\right) c_{d a}^{s}} \exp \left(-\frac{\gamma \beta c_{0}}{k T}\right)-\frac{\gamma \beta}{k T} J\left(c_{d a}^{s}\right) \\
& +\int_{c_{0}}^{c_{d a}^{s}} \frac{d c_{d}}{\left(1-c_{d}\right)^{2}\left(-c_{d}+c_{a d}^{s}+c_{0}\right)} \exp \left\{-\frac{\gamma \beta c_{d}}{k T}\right\} .
\end{aligned}
$$

\section{4. Макропластичность}

Синергетика динамических дислокационной и примесной подсистем проявляется в доступных наблюдению макроскопических процессах пластической деформации кристаллов. Будем находить скорость пластического течения $\dot{\varepsilon}$ с помощью закона Орована, $\dot{\varepsilon}=\rho b V$, в котором плотность дислокаций $\rho$ приближенно считается постоянной. Здесь $b-$ величина вектора Бюргерса дислокаций. Выражая скорость дислокации через $\dot{\varepsilon}$, преобразуем уравнение (11) к виду

$$
\frac{\dot{\varepsilon}}{\rho b}=\frac{a}{\tau} \frac{1-c_{d a}^{s}-c_{0}}{\ln \left\{\left(1-c_{d a}^{s}\right) c_{d a}^{s} /\left[\left(1-c_{0}\right) c_{0}\right]\right\}} .
$$


Это уравнение вместе с уравнением (10) составляют систему, из которой зависимость напряжения от скорости деформации находится самосогласованным образом.

Экспериментальный активационный объем определяется как

$$
\gamma_{\exp }=\frac{k T}{d \sigma / d \ln \dot{\varepsilon}}
$$

где $d \sigma / d \ln \dot{\varepsilon}=V d \sigma / d V$ и $d \sigma / d V$ дается выражением (12). Границе существования области неустойчивости дислокационной подвижности соответствует обращение скоростной чувствительности в нуль, что приводит к пикам эффективного активационного объема, в размытом виде наблюдаемым экспериментально [3].

\section{5. Обсуждение результатов}

Наличие примесей в ядре из-за зацепления примесей за кристаллическую решетку тормозит движение дислокации. В результате, чтобы получить ту же самую скорость дислокации, например, при пластическом деформировании, требуется увеличение прикладываемой движущей силы, что свидетельствует об упрочнении материала. Дополнительный вклад в движущую силу будет отражать поведение примесного содержания в ядре при изменении различных параметров. При медленном движении дислокаций содержание примесей в их ядрах близко к равновесному и поведение силы пиннинга аналогично тому, что наблюдается при статическом старении дислокаций: сила пиннинга убывает с ростом температуры. Специфика динамического старения дислокаций проявляется при достаточно быстром их движении и обусловлена показанным в настоящей работе наличием особой начальной стадии в кинетике перераспределения примесей вокруг дислокационного ядра. Предсказываемый рост содержания примесей обусловливает различного типа аномалии, которые неоднократно наблюдались в экспериментальных работах во многих материалах: в металлах с ОЦК структурой Mo, V и др., в разупорядоченных твердых растворах, при призматическом скольжении в металлах с ГПУ-структурой $\mathrm{Ti}, \mathrm{Zr}, \mathrm{Be}$ и интерметаллидах (см. обзор в [3]).

Следует отметить, что для проявления этих особенностей имеет значение не столько средняя по образцу концентрация примесей $c_{0}$, сколько избыточная концентрация в ядре дислокации $c_{d}$, которая, в зависимости от температуры, меняется в широких пределах вплоть до насыщения $c_{d} \rightarrow 1$. Достаточно большое увеличение содержания примесей в ядрах способно понизить подвижность дислокаций ниже наблюдаемого предела, приводя фактически к иммобилизации дислокаций [30] или к охрупчиванию материала $[23,24]$. В этой связи предсказываемый уравнением (11) сильный рост концентрации может являться причиной такого интересного явления, как „инверсный хрупко-пластический переход“, при котором материал теряет свою пластичность не с понижением температуры как обычно, a, напротив, с ее повышением, как это наблюдалось в оливине [31], $\mathrm{GaAs}$ [32] или $\mathrm{SrTiO}_{3}$ [33].

\section{6. Заключение}

Как показывают результаты проведенных расчетов, предлагаемая модель содержит достаточный потенциал, чтобы выявить причины и описать механизм аномального поведения пластической деформации кристаллических материалов. В то же время в ней не учитываются многочисленные процессы, которые могут существенно проявляться на более поздних стадиях, например, длиннопробежная диффузия примесей или какая-либо специфика конкретных материалов. Эти процессы необходимы для описания выхода из аномального режима, но мало влияют на его возникновение. Потому при описании условий возникновения аномалий можно ограничиться развиваемой упрощенной моделью, достоинством которой является ее универсальность.

Отметим основные результаты исследования.

1. Сформулирована модель динамического старения быстро движущихся дислокаций в примесных материалах с высоким кристаллическим рельефом, когда континуальное описание примесных атмосфер неприменимо.

2. Показано наличие стадии динамического старения дислокаций с образованием существенно неравновесного содержания примесей вблизи ядра дислокации.

3. Рассчитаны кинетические закономерности динамического старения дислокаций, обосновано отличающиеся от произвольно постулируемых в классической теории.

4. Показано, что наличие стадии первоначального быстрого роста примесного содержания в дислокационном ядре является механизмом аномальных отрицательной скоростной чувствительности пластического течения материалов и позитивной температурной зависимости деформирующего напряжения.

\section{Финансирование работы}

Работа выполнена при поддержке Министерства науки и высшего образования в рамках выполнения работ по Государственному заданию ФНИЦ „Кристаллография и фотоника" РАН.

\section{Конфликт интересов}

Автор заявляет, что у него нет конфликта интересов.

\section{Список литературы}

[1] Г.А. Малыгин. ФТТ 60, 6, 1232 (2018).

[2] Ж. Фридель. Дислокации. Мир, М. (1967). 643 с.

[3] D. Caillard, J.L. Martin. Thermally Activated Mechanisms in Crystal Plastisity. Pergamon, Amsterdam-Boston-London-N.Y.-Oxford-Paris-San Diego-San Francisco-Singapore-Sidney-Tokyo (2003). 433 p.

[4] U. Messerschmidt. Dislocation Dynamics During Plastic Deformation / Ed Hull R. Berlin. Heidelberg: Springer Series in Material Science (2010). 
[5] Б.В. Петухов. Динамика дислокаций в кристаллическом рельефе. Дислокационные кинки и пластичность кристаллических материалов. Saarbrücken: Lambert Academic Publishing. (2016). 385 p.

[6] B.V. Petukhov. J. Physics: Conf. Ser. 240, 012012 (2010).

[7] A. Portevin, F. Le Chatelier. Trans. Am. Soc. St. Tr. 5, 457 (1924).

[8] P.G. McCormick. Acta Met. 20, 3, 351 (1972).

[9] A. van den Beukel. Phys. Status Solidi A 30, 1, 197 (1975).

[10] G. Ananthakrishna. Phys. Rep. 440, 4-6, 113 (2007).

[11] Y. Estrin, L.P. Kubin. In: Continuum Models for Materials with Microstructure / Ed. Mühlhaus H.B. Chichester. Wiley (1995). P. 395.

[12] T.S. Gross, V.K. Mathews, R.J. De Angelis, K. Okazaki. MSE A117, 9, 75 (1989).

[13] A.H. Cottrell, M.A. Jaswon. Proc. Roy. Soc. A. 199, 1056, 104 (1949).

[14] H. Yoshinaga, S. Morozumi. Phil. Mag. 23, 186, 1351 (1971).

[15] G.A. Malygin. Phys. Status Solidi A 72, 2, 493 (1982).

[16] Y. Wang, D.J. Srolovitz, J.M. Rickman, R. Lesar. Acta Mater. 48, 9, 2163 (2000).

[17] D. Maroudas, R.A. Brown. J. Mater. Res. 6, 11, 2337 (1991).

[18] W.A. Curtin, D.L. Olmsted, L.G. Hector. Jr. Nature Mater. 5, 11, 875 (2006).

[19] N. Louat. Scr. Met. 15, 11, 1167 (1981).

[20] Дж. Хирт, И. Лоте. Теория дислокаций. Атомиздат, М. (1972). $598 \mathrm{c}$.

[21] B.V. Petukhov. Phys. Status Solidi 2, 6, 1864 (2005).

[22] S.C. Park, L.P. Beckerman, R.E. Reed-Hill. Metallurg. Trans. A 14, 3, 463 (1983).

[23] M. Itakura, H. Kaburaki, M. Yamaguchi, T. Okita. Acta Mater. 61, 18, 6857 (2013).

[24] P. Gong, I.H. Katzarov, J. Nutter, A.T. Paxton, W.M. Rainforth. Sci. Rep. 10, 10209 (2020).

[25] Y. Zhao, L. Dezerald, M. Pozuelo, X. Zhou1, J. Marian. Nature Commun. 11, 1227, 1 (2020).

[26] Б.В. Петухов. Физ. мет. и металловедение 56, 6, 1177 (1983).

[27] B.V. Petukhov. Mater. Sci Eng. A. 234-236, 177 (1997).

[28] B. Petukhov. Electron. Proc. MRS Spring Meeting, 683E, BB3.3 (2001).

[29] B. Lüthi, L. Ventelon, D. Rodney, F. Willaime. Comput. Mater. Sci. 148, 1, 21 (2018).

[30] I.E. Bondarenko, V.G. Eremenko, V.I. Nikitenko, E.B. Yakimov. Phys. Status Solidi A 60, 2, 341 (1980).

[31] H.W. Green II, T.E. Young, D. Walker, C.H. Scholz. Nature 348, 12, 720 (1990).

[32] T. Suzuki, T. Tokuoka, I. Yonenaga, H.O.K. Kirchner. Scripta Mater. 43, 7, 645 (2000).

[33] P. Gumbsch, S. Taeri-Baghbadrani, D. Brunner, W. Sigle, M. Rühle. Phys. Rev. Lett. 87, 085505 (2001).

Редактор Т.Н. Василевская 